\title{
Imaging and Elastometry of Blood Clots Using Magnetomotive Optical Coherence Tomography and Labeled Platelets
}

\author{
Amy L. Oldenburg, Gongting Wu, Dmitry Spivak, Frank Tsui, Alisa S. Wolberg, and Thomas H. Fischer
}

(Invited Paper)

\begin{abstract}
Improved methods for imaging and assessment of vascular defects are needed for directing treatment of cardiovascular pathologies. In this paper, we employ magnetomotive optical coherence tomography (MMOCT) as a platform both to detect and to measure the elasticity of blood clots. Detection is enabled through the use of rehydrated, lyophilized platelets loaded with superparamagnetic iron oxides (SPIO-RL platelets) that are functional infusion agents that adhere to sites of vascular endothelial damage. Evidence suggests that the sensitivity for detection is improved over threefold by magnetic interactions between SPIOs inside RL platelets. Using the same MMOCT system, we show how elastometry of simulated clots, using resonant acoustic spectroscopy, is correlated with the fibrin content of the clot. Both methods are based upon magnetic actuation and phase-sensitive optical monitoring of nanoscale displacements using MMOCT, underscoring its utility as a broad-based platform to detect and measure the molecular structure and composition of blood clots.
\end{abstract}

Index Terms-Elasticity, magnetic forces, nanoparticle, optical imaging, thrombosis.

\section{INTRODUCTION}

B LOOD clotting is the human body's way of preventing excessive bleeding, while at the same time, inappropriate clotting at sites of vascular damage is the source of cardiovascular "events" and the driver of thrombotic complications. The detection of clots and characterization of clot function is,

Manuscript received May 21, 2011; revised July 12, 2011; accepted July 12 , 2011. Date of publication July 22, 2011; date of current version June 1, 2012. This work was supported in part by the U.S. Department of Defense under Grant ONR N00014-10-1-0792 to ALO, the National Institutes of Health under Grant R01HL094740 to ASW, NC TraCS UL1RR025747 with pilot Grants R61022 to ALO and R81034 to ASW), and startup funds at the University of North Carolina at Chapel Hill (ALO). Additional information can be found at http://www.physics.unc.edu/ aold/index.html

A. L. Oldenburg is with the Department of Physics and Astronomy and the Biomedical Research Imaging Center, University of North Carolina at Chapel Hill, Chapel Hill, NC 27599-3255 USA (e-mail: aold@physics.unc.edu).

G. Wu, D. Spivak, and F. Tsui are with the Department of Physics and Astronomy, University of North Carolina at Chapel Hill, Chapel Hill, NC 27599-3255 USA (e-mail: gwu@email.unc.edu; mitja@email.unc.edu; ftsui@ physics.unc.edu).

A. S. Wolberg and T. H. Fischer are with the Department of Pathology and Laboratory Medicine, University of North Carolina at Chapel Hill, Chapel Hill, NC 27599-7525 USA (e-mail: alisa_wolberg@med.unc.edu; tfischer@med.unc.edu).

Color versions of one or more of the figures in this paper are available online at http://ieeexplore.ieee.org.

Digital Object Identifier 10.1109/JSTQE.2011.2162580 therefore, of paramount medical importance. Currently, optical coherence tomography (OCT) is gaining use as an intravascular imaging modality that provides high-resolution details of vascular lesions, particularly in the coronary artery for the identification of unstable plaques [1]-[3], assessing vascular conduit quality during bypass surgery [4], and monitoring balloon inflation during angioplasty [5]. OCT image contrast is based upon tissue backscattering, which provides only limited information about vascular structure. New OCT-based methods to specifically contrast blood clots and their mechanical properties have the potential to greatly expand the clinical utility of OCT.

One of the most advanced techniques for OCT contrast imaging is based upon the use of magnetic nanoparticles, specifically superparamagnetic iron oxides (SPIOs), that are magnetically actuated during OCT imaging [6]-[8]. Application of a modulated magnetic field using external electromagnets causes periodic magnetically induced motion (magnetomotion) of SPIOs, which is subsequently detected with OCT through a process dubbed magnetomotive OCT (MMOCT). MMOCT has been employed in a variety of applications, including in vivo molecular imaging for detection of cancer [9], monitoring diffusion of SPIOs [8], and tracking SPIO-labeled cells [6]. Because tissue magnetomotion is dictated by the tissue elastic properties, elastography using MMOCT, or magnetomotive optical coherence elastography (MMOCE), is also a growing application [10]. In particular, using MMOCE to measure resonances in the acoustic spectrum of soft tissues has the potential to be a very accurate method for tissue sample elastometry [11].

For the purposes of contrasting blood clots with MMOCT, combining SPIOs with platelets is significant because of the capability of these cells to localize to inflamed and injured vasculature in a wide range of pathologies. The pathophysiological function of platelets is to detect and adhere to sites of vascular damage for the formation of primary hemostatic plugs, and then to amplify coagulation reactions so as to limit hemorrhage [12]. As such, SPIO-labeled platelets have broad-based potential for detection of localized vascular endothelial defects in cardiovascular disease and trauma.

Rehydrated, lyophilized platelets (RL platelets) are used in this study as an infusion agent that are prepared from fresh human apheresis platelets and overcome difficulties with platelet storage and harvesting [13]. Recently, the development of SPIORL platelets as a functional MMOCT imaging agent has been reported [14]. In that study, we found that SPIO-RL platelets 
maintained their native hemostatic platelet ability to specifically adhere to damaged vascular endothelium ex vivo, providing the ability to distinguish between injured and uninjured endothelium with MMOCT imaging. While the demonstrated detection sensitivity to SPIO-RL platelets incorporated into blood clots is feasible for in vivo use; here, we will show how control of SPIO-RL platelet magnetic properties can further increase imaging sensitivity, which will lower the dose needed in realistic imaging applications.

At the same time, blood clot elasticity is a potentially powerful metric for predicting the risk associated with the presence of blood clots. Abnormal clot elasticity is associated with myocardial infarction [15], coronary atherothrombosis [16], ischemic stroke [17], venous thromboembolism [18], and diabetes [19]. The importance of clot elastic modulus is evidenced by the growing use of devices such as the thromboelastograph (TEG) and the Hemodyne [20]. As we will show later, MMOCE has the potential to enhance the accuracy of clot elastometry by the use of resonant acoustics, while using the same imaging hardware for the detection of SPIO-RL platelets in clots.

\section{MMOCT}

Here, we briefly review the relevant background and capabilities of MMOCT, for which detailed theoretical analyses can be found elsewhere [7], [8]. OCT is a favorable platform for imaging nanoscale tissue displacements such as those induced by SPIOs. OCT is based upon interferometric detection of backscattered, low-coherence light to optically depth range tissue structure up to several mean free scattering path lengths deep [21], [22]. Spectral-domain OCT provides excellent phase (and hence displacement) sensitivity by avoiding the need for a moving delay line; this is accomplished by collecting the spectral interferogram and relating it to the time-domain signal through Fourier transformation [23]. While the phase sensitivity of our OCT system corresponds to a displacement (jitter) noise of $\sim 11 \mathrm{~nm}$, subnanometer phase sensitivities have been reported [24].

The ability to detect the presence of SPIOs in tissue using OCT and magnetically induced motion (MMOCT) can be understood in several steps. The application of a gradient magnetic field $B$ to tissue containing SPIOs results in a local magnetic gradient force $F$ per volume $V$ according to

$$
\frac{F}{V}=\left(\left(M_{p}+M_{d}\right) \cdot \nabla\right) B
$$

where $M_{p}$ and $M_{d}$ are the volume magnetizations arising from the SPIOs and the tissue, respectively. While the magnetization from SPIOs is positive (paramagnetic), the magnetization from tissue is negative (diamagnetic). As such, the net force between the paramagnetic and diamagnetic components of the tissue sample can point in either direction. MMOCT operates by rejecting the negative (diamagnetic) tissue motion to avoid false positives; therefore, a sufficient concentration of SPIOs is needed to make the force net positive. Because SPIOs have a magnetic susceptibility $>10^{5}$ times larger than that of tissue, the net positive force requirement sets the threshold concentration of SPIOs for detection to be $<10^{-5}$ fractional volume loading in

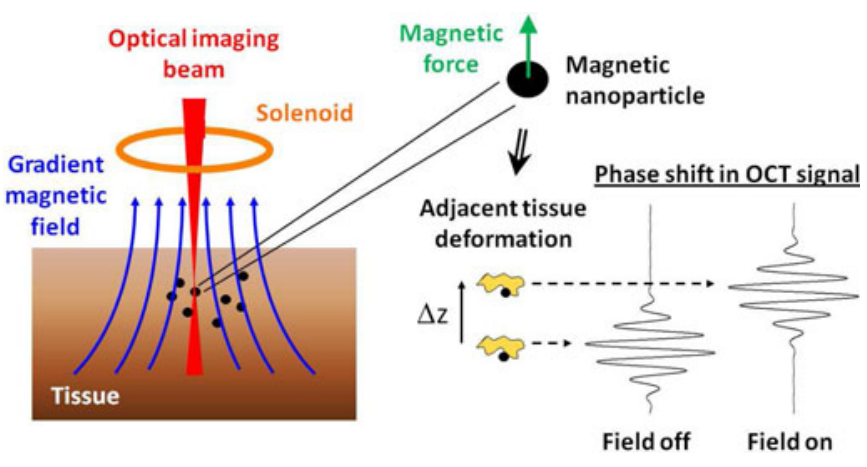

Fig. 1. Cartoon schematic of the principle of MMOCT depicting how a magnetic actuation of nanoparticles inside the imaging volume induces a phase shift in the OCT interferogram.

tissue. Counterintuitively, the use of higher magnetic fields that saturate the SPIOs act to raise this minimum threshold, because the diamagnetic components continue to increase linearly [8]. This results in an optimal magnetic field of typically $0.1-0.2 \mathrm{~T}$, and due to the small imaging volume associated with OCT, only a small, water-jacketed electromagnet is needed to be placed above the tissue during imaging (see Fig. 1).

These magnetic gradient forces act collectively on all SPIOs in the tissue volume, resulting in net body forces inside the tissue that are proportional to the local concentration of SPIOs. The tissue then elastically deforms in response to mechanical coupling with the SPIOs. While SPIOs themselves are light absorbing and undetectable, the tissue deformation is directly detected by OCT. Specifically, the optical phase shift observed in the OCT interferogram $\Delta \phi$ corresponds to the axial tissue displacement $\Delta z$ according to $\Delta \phi=4 \pi \Delta z / \lambda$. In our MMOCT system, we preferentially induce axial displacements by centering a solenoid coil on-axis with the imaging beam (see Fig. 1).

The amount of tissue displacement is both proportional to the concentration of SPIOs and inversely proportional to the elastic modulus of the tissue; as such, it is not currently possible to extract a quantitative measurement of one without a priori knowledge of the other.

The most important feature of MMOCT is the ability to modulate the magnetic gradient force at a frequency that rejects other sources of noise, particularly tissue movements arising from respiration and cardiac function. Bandpass filtering of the optical phase signal is employed around the magnetic modulation frequency, and the addition of a filter based on the relative phase of the magnetomotion (to reject diamagnetic motion) has resulted in sensitivities as low as $27 \mu \mathrm{g} / \mathrm{g}$ [8]. This combination of sensitivity, specificity, and speed has recently enabled a variety of novel studies for tracking SPIOs in vivo [7], [9].

\section{MMOCE}

Because MMOCT relies on the detection of elastic tissue deformation, it is also possible to use it in systems with controlled boundary conditions for elastography (MMOCE) [10], [11]. Of special interest is the measurement of small biosamples which can be made to mechanically resonate at low $(<1 \mathrm{kHz})$ frequencies. Elastometry of such small $\left(<10 \mathrm{~cm}^{3}\right)$, soft 


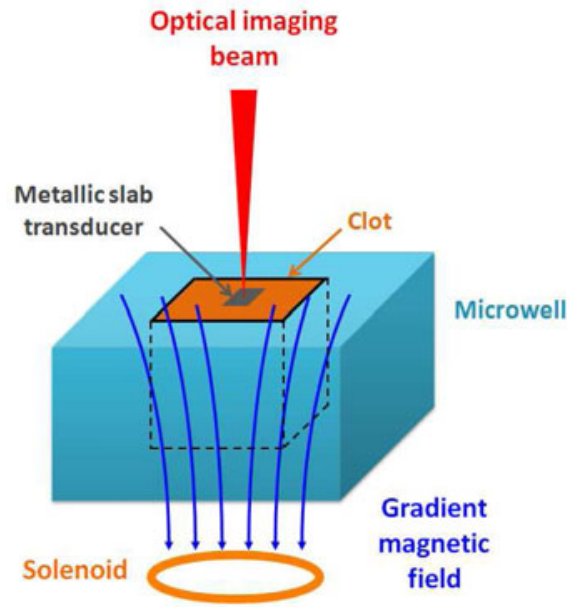

Fig. 2. Cartoon schematic of MMOCE depicting the geometry of clot elastometry performed in a rectangular microwell.

$(<100 \mathrm{kPa})$ biosamples, such as blood clots, is difficult to accomplish by traditional methods due to large error arising from ambiguity with the actuator contact position, and due to the need for extremely low actuation forces, low actuator inertia, and nanoscale displacement sensitivity. MMOCE mediates these difficulties by providing magnetic control of forces, optical phase detection, and micro- and nanotransducers that are already in contact with the tissue and impart negligible inertia.

The reason that small, soft biosamples in particular can be made to mechanically resonate is due to elastic wave reflections from their lower boundary that set up standing longitudinal waves with wavelengths $\lambda_{n}$ of mode number $n$ as allowed by the boundary conditions. The resonance frequency $\omega_{n}$ associated with each longitudinal mode $n$ can be written generally as [11]

$$
\omega_{n}=\frac{2 \pi}{\lambda_{n}} g \sqrt{\frac{E}{\rho}}
$$

where $E$ is the elastic modulus of the tissue, $\rho$ is the mass density, and $g$ is a geometrical factor that depends only upon $n$, the boundary conditions, sample geometry, and Poisson's ratio. In a previous study [11], it was shown that longitudinal resonances in cylinders with open sides could be predicted by calculating $g$ according to elastic theory [25]. In this study, we will focus on the relative changes in $E$ for fibrin clot samples with closed, rectangular sides by monitoring $\omega_{0}$ and assuming Poisson's ratio, and therefore $g$, and $\lambda_{0}$, are constant. In practice, samples with controlled boundary conditions can be obtained by solidifying a liquid sample (e.g., blood) into a well of defined shape, cutting a solid tissue sample with a punch as in [26], or by using the OCT system itself to image the shape of the tissue sample. Another practical simplification we introduce here for clot elastometry (see Fig. 2) is the use of a microtransducer placed on top of the sample, instead of embedded SPIOs. This avoids difficulties with the interference that SPIOs have on the clot formation, chemistry, and mechanical properties.
The nanoscale displacement sensitivity afforded by OCT translates to typically microstrain sample sensitivity. These perturbative strains allow for measurements of tissue in the linear viscoelastic regime, where the displacement of the system under time-harmonic excitation, $\Delta z(\omega)=\tilde{A}(\omega) e^{i \omega t}$, can be written in terms of the complex amplitude $\tilde{A}(\omega)$ as a Lorentzian:

$$
\tilde{A}(\omega)=\frac{q}{\omega_{n}^{2}-\omega^{2}-i \omega \gamma}
$$

where $\gamma$ is the damping coefficient and $q$ is proportional to the driving force. In our implementation of MMOCE, a chirped (frequency-swept) sinusoidal magnetic force is applied to measure the acoustic spectrum of a tissue sample. Chirping provides a measurement of the system impulse response and has a signalto-noise advantage over the use of a mechanical impulse because the measurement is spread out over a longer acquisition time. It is important to note that we previously found that tissues tend to be underdamped [11], with a quality factor $Q \approx 3$ for rat liver, such that the amplitude of magnetomotion at the acoustic resonance is significantly amplified.

\section{METHODS}

\section{A. Preparation of SPIO-RL Platelets in Clot-Like Aggregates}

Here, we describe the preparation of SPIO-RL platelet aggregates for a comparative study with the dispersed SPIO-RL platelets in our previous study [14]. In this study, dextran-coated SPIOs were prepared with the method of Molday and MacKenzie [27] at an iron concentration of $0.91 \mathrm{mg} / \mathrm{ml}$ in citrated saline ( $5.375 \mathrm{mM}$ citrate, $165 \mathrm{mM} \mathrm{NaCl}, \mathrm{pH}=7.4$ ). This method is expected to produce dextran-coated SPIOs with core sizes of $10-20 \mathrm{~nm}$, outer diameter of $30-40 \mathrm{~nm}$, and $50 \% \mathrm{Fe}$ content by weight. In comparison, in our previous study Feridex I.V. (Advanced Magnetics) was used which is also a dextran-coated SPIO with core size $\sim 5 \mathrm{~nm}$, number-weighted hydrodynamic diameter of $36 \mathrm{~nm}$ [28], and $\sim 60 \% \mathrm{Fe}$ content by weight (computed from the concentrations reported on the package insert).

SPIO-RL platelets were prepared by incubating an equal volume of prepared SPIOs with one-day-old apheresis platelets at a concentration of $1.1 \times 10^{9} / \mathrm{cm}^{3}$. SPIO platelet aggregates were then separated from single platelets with internalized SPIO and free extracellular SPIO with differential sedimentation velocity and density gradient centrifugation as described elsewhere [29] and then subjected to aldehyde stabilization and lyophilization [13]. Examination with light microscopy showed that after rehydration, the SPIO-RL platelets were present as aggregates with a structure typical of primary hemostatic clots.

The main difference between the preparation used here compared to that reported earlier [14] involves how the platelets were separated from excess SPIO. Size exclusion chromatography was utilized to separate platelets from extracellular SPIO in the earlier study. This produced SPIO-containing platelets with an activated nondiscoidal morphology with extended pseudopodia, but that were largely unaggregated. In contrast, when excess SPIO was separated from platelets on the bases of sedimentation velocity and density with centrifugation, an aggregated population of platelets with activation and associative properties 
similar to platelets in primary hemostatic clots was obtained. The platelets were present in aggregates of ten to several hundred with closely juxtaposing membranes, showed signs of cytoskeletal rearrangement, and granule secretion. These results demonstrate that care will be required to prepare infusible SPIORL platelets for potential human applications.

\section{B. Preparation of Fibrin Clots}

Simulated clots composed of fibrin were prepared to investigate the relationship between fibrin content and clot elasticity. Fibrin clots were made by mixing $0.3-3.7 \mathrm{mg} / \mathrm{mL}$ (final concentration) plasminogen-, fibronectin-, and von Willebrand factor-depleted human fibrinogen (Enzyme Research Laboratories, South Bend, IN) with $25 \mathrm{nM}$ (final concentration) human thrombin (Haematologic Technologies, Essex Junction, VT) in $20 \mathrm{mM}$ N-2-hydroxyethylpiperazine- $\mathrm{N}^{\prime}$-2-ethanesulfonic acid (pH 7.4), $150 \mathrm{mM} \mathrm{NaCl}, 1 \mathrm{mg} / \mathrm{mL}$ bovine serum albumin, and $5 \mathrm{mM} \mathrm{CaCl}_{2}$ at room temperature. Clots were formed inside custom-printed rectangular wells of $6 \mathrm{~mm} \times 6 \mathrm{~mm} \times 10 \mathrm{~mm}$ (length $\times$ width $\times$ depth) dimensions in a humidified chamber, and MMOCE was performed after fibrin clots had fully formed ( $60 \mathrm{~min})$.

\section{Magnetic Properties: Measurements and Modeling}

We employed superconducting quantum interference device (SQUID) magnetometry to measure the magnetic properties of SPIOs and SPIO-RL platelet aggregates. A 5 T SQUID magnetometer (Quantum Design) was employed for this study. SPIOs (at $22.5 \mu \mathrm{g} \mathrm{Fe} / \mathrm{mL}$ ) and SPIO-RL platelets (at $65 \mu \mathrm{g} \mathrm{Fe} / \mathrm{mL}$ ) were dispersed into $1 \%$ agarose and sealed in a plastic capsule for analysis. In addition, SPIO-RL platelets were analyzed in anticoagulated fresh porcine blood, and in clotted porcine blood (by addition of $11 \mathrm{mM} \mathrm{CaCl} 2$ ), both at a concentration of $41-43 \mu \mathrm{g} \mathrm{Fe} / \mathrm{mL}$. Porcine blood was drawn from healthy swine according to approved protocols of the Institutional Animal Care and Use Committee at the University of North Carolina at Chapel Hill. Magnetization versus field $M(H)$ hysteresis loops were measured at $20^{\circ} \mathrm{C}$. Calibration and subtraction of the diamagnetic contribution from the agarose, blood, and plastic capsule was performed as described previously [14]. Then, the saturation magnetization $M_{s}$ and an effective magnetic moment $\mu$ were used as free-fitting parameters in the Langevin function given by

$$
M(H)=M_{s}\left(\operatorname{coth}\left(\frac{\mu H}{k_{B} T}\right)-\frac{k_{B} T}{\mu H}\right)
$$

where $k_{B}$ is Boltzmann's constant, $T$ is the temperature, and the effective moment $\mu$ can be expressed in numbers of Bohr magnetons $\mu_{B}$. We note that $\mu$ can be interpreted as the effective size of the superparamagnetic spin cluster and is proportional to $\chi / M_{s}$ and thus independent of the amount of material (either mass or volume) in the sample, where $\chi$ is the low-field magnetic susceptibility. As such, $\mu$ is an important parameter that determines the low-field susceptibility and magnetization, and consequently the magnetically induced force (1), at low fields like those used in MMOCT.
In order to further examine the magnetic interactions and the effective moment of SPIOs and SPIO-RL platelets, temperaturedependent field-cooled (FC) and zero-field-cooled (ZFC) dc magnetization and ac susceptibility measurements were performed. The ZFC measurement entailed first cooling to $5 \mathrm{~K}$ in nominal zero field $(<1 \mathrm{Oe})$, applying a 50 -Oe field, and then measuring the $\mathrm{dc}$ and ac magnetic responses as the temperature was warmed to $320 \mathrm{~K}$. The FC scan was subsequently performed in 50 Oe as the temperature was cooled from 320 back to $5 \mathrm{~K}$. $\mathrm{AC}$ susceptibility was performed with a field modulation frequency of $10 \mathrm{~Hz}$ and amplitude of $4 \mathrm{Oe}$. At temperatures above the onset of irreversibility, the measured low-field magnetization and susceptibility were, respectively, fit to the Curie law according to

$$
\begin{aligned}
M(T) & =\frac{\mu M_{s}}{3 k_{B} T} H \quad \text { and } \\
\chi(T) & =\frac{\Delta M(T)}{\Delta H}=\frac{\mu M_{s}}{3 k_{B} T} .
\end{aligned}
$$

Here, the value for $M_{s}$ from field-dependent measurements was used, and the effective moment $\mu$ was the only fitting parameter.

\section{Methods for MMOCT and MMOCE}

The MMOCT methods used for detection and imaging of SPIO-RL platelet aggregates in tissue phantoms are identical to those described in detail previously [14]. Briefly, a custom spectral-domain OCT system comprised of a Ti:Sapphire laser source centered at $810 \mathrm{~nm}$ with $\sim 125$-nm bandwidth (Griffin, Kapteyn-Murnane Labs, Boulder, $\mathrm{CO}$ ) and use of a $30-\mathrm{mm}$ focal length imaging lens provided $3 \times 12 \mu \mathrm{m}$ (axial $\times$ transverse) imaging resolution and $\sim 10 \mathrm{~mW}$ of optical power at the sample. Images were collected into successive B-mode $(x-z)$ frames over $2500 \times 9 \times 1024$ pixels $(x \times y \times z)$ at a linerate of $1 \mathrm{kHz}$ with a charge-coupled device camera (Dalsa Piranha 2) during magnetic field modulation at $100 \mathrm{~Hz}$. The peak magnetic field and gradient were $0.15 \mathrm{~T}$ and $15 \mathrm{~T} / \mathrm{m}$, respectively. Reconstruction of OCT and MMOCT images was performed according to a phase-resolved method described previously using bandpass filtering and removal of induced motions from the diamagnetic tissue background [8].

MMOCE was performed using the same system hardware for elastometry of fibrin clots. General methods for MMOCE using resonant acoustic spectroscopy have been described in detail previously [11]. The primary difference between this and previous work is our use of a microtransducer placed onto the top surface of the clot, instead of embedding clots with SPIOs. However, care is needed to avoid the unwanted perturbation of the system inertia by the use of a microtransducer with mass much smaller than that of the sample. In our experiments, the microtransducer consisted of a $2 \mathrm{~mm} \times 2 \mathrm{~mm} \times 0.025 \mathrm{~mm}$ amorphous steel alloy sheet wrapped with tape to provide diffuse optical scattering; its mass was $\sim 2 \mathrm{mg}$, which is less than $1 \%$ of our sample mass of $360 \mathrm{mg}$.

Experiments were performed by tracking the axial displacement of the microtransducer with the OCT system in M-mode (depth at one transverse position versus time) at a linerate of 
$2 \mathrm{kHz}$, while sweeping the magnetic field modulation rate from 0 to $400 \mathrm{~Hz}$. In these experiments, the solenoid was placed beneath the sample to provide a downward force onto the microtransducer. Furthermore, the voltage was decreased to prevent motion that would be too rapid for detection by the OCT system (avoiding phase wrapping during the period of line acquisition); the resulting magnetic field was, therefore, much smaller than 0.15 T. M-mode images were collected into $4000 \times 1024$ pixels (time $\times$ depth). Image processing was performed to extract the amplitude and phase of the acoustic spectrum by dividing the optical phase data by the chirped waveform of the magnetic force, as described in detail previously [11]. The position of the fundamental resonant frequency was obtained by separately fitting the amplitude (absolute value) and phase (argument) of the displacement spectrum of (3), with $\omega_{0}$ and $\gamma$ as fitting parameters, and computing the average value of $\omega_{0}$. Two scans were obtained for each sample.

\section{RESUlTS AND Discussion}

\section{A. Ex Vivo Imaging of Blood Clots Using MMOCT and SPIO-RL Platelets in Porcine Arteries}

In this section, we briefly review our previous results using SPIO-RL platelets to provide specific imaging contrast to damaged vascular endothelium. This will be a point of comparison for the new SPIO-RL platelets analyzed in this study. We found that each platelet could be loaded with $48.3 \pm 0.6 \mathrm{fg}$ Fe (by mass spectrometry) from SPIOs comprised of Feridex I.V. The SPIOs in each SPIO-RL platelet, then, act as a magnetic handle by which to contrast the platelets. At these loading levels, we found that the magnetic properties of SPIO-RL platelets predict a threshold concentration of $0.27 \times 10^{9} / \mathrm{cm}^{3}$ for MMOCT contrast, and that, in practice, SPIO-RL platelets were detected at concentrations $\geq 1.5 \times 10^{9} / \mathrm{cm}^{3}$. Assuming an average platelet volume of $10.6 \mathrm{fL} \mathrm{[30],} \mathrm{this} \mathrm{latter} \mathrm{corresponds} \mathrm{to} \mathrm{a} \mathrm{volume}$ fraction of only $1.6 \%$. In comparison, microscopic examination of arterial thrombi reveals that native platelets can occupy a significant fraction of the thrombus [31].

Our most important finding is that SPIO-RL platelets maintained their native platelet ability to specifically adhere to injured arteries, thus acting as contrast agents for MMOCT-based detection of blood clots [14]. Briefly, ex vivo porcine arteries were injured with a sharp steel cytology brush and ligated at one end with a catheter for sample infusion. A mixture of normal porcine blood containing the endogenous porcine platelets and SPIORL platelets was infused over a 4 -min period at $0.25 \mathrm{~mL}$ blood mixture/min. After exposure, arteries were saline flushed and images were obtained before and after opening the arteries. One out of two artery segments showed significantly large MMOCT signal, with an image-averaged signal exceeding $3.2 \mathrm{~dB}$, compared to $\leq 0.12 \mathrm{~dB}$ for control.

Fig. 3 displays a representative set of MMOCT images for damaged and undamaged arteries. Note that no MMOCT signal is obtained in the absence of magnetic field modulation, as expected. Importantly, no significant MMOCT signal is obtained in a control (undamaged) blood vessel that was exposed to the same concentration of SPIO-RL platelets. This suggests

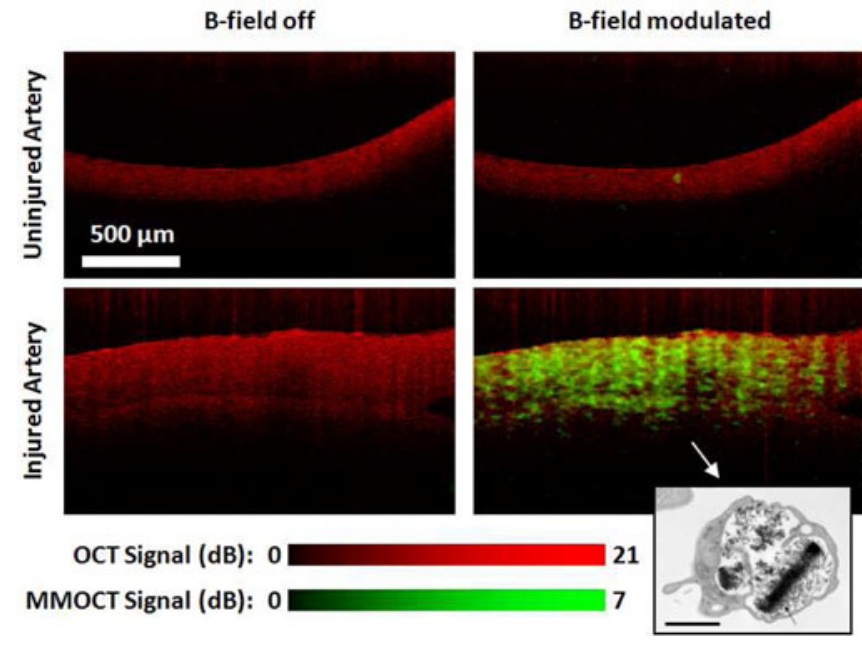

Fig. 3. OCT (red) and MMOCT (green) of ex vivo porcine arteries exposed to SPIO-RL platelets in a flow chamber. MMOCT contrast is specific to the artery that was injured, due to specific adhesion of SPIO-RL platelets. Lower right inset: TEM of an SPIO-RL platelet, $1 \mu \mathrm{m}$ scalebar.

that platelets, after preparation into SPIO-RL platelets, retained the ability to distinguish injured from uninjured endothelium. This is consistent with earlier evidence that the hemostatic function of RL platelets is mediated by activatable primary hemostatic mechanisms in combination with activation of the cell membrane to catalyze coagulation cascade turnover [32]. Future study of SPIO-RL platelets is needed to determine whether the introduction of SPIOs modify these hemostatic mechanisms.

\section{B. Improved Magnetic Properties of SPIO-RL Platelets Lead to Higher Sensitivity in MMOCT}

While the previous results for blood clot detection by MMOCT of SPIO-RL platelets are promising, further reduction of the detection threshold would relax the practical requirement upon the dose of SPIO-RL platelets needed for blood clot detection. This can be accomplished by improving the magnetic properties of the SPIO-RL platelets. In this study, SPIO-RL platelet aggregates were prepared which contained $>4 \times$ the amount of Fe per platelet (see Table I). Furthermore, their activation and hemostatic clot-like formation may approximate the in vivo environment of SPIO-RL platelets that have adhered to a blood clot. As such, we measured and compared the magnetic and MMOCT contrast properties of the SPIO-RL platelet aggregates to the previous SPIO-RL platelets, which are summarized in Table I.

It is important to note which magnetic properties are most effective for enhancing MMOCT contrast. According to (1), the magnetically induced force is dictated by the difference in magnetization of the SPIOs from that of the diamagnetic medium around the SPIOs. As shown previously [8], this actually dictates the use of smaller magnetic fields (typically $0.1-0.2 \mathrm{~T}$ ) that do not fully saturate the SPIOs, in order to minimize the deleterious effects of the diamagnetic medium and to optimize the MMOCT detection threshold. The ideal SPIO-RL platelet, 
TABLE I

Magnetic and MMOCT Contrast Properties of Free SPIOS AND SPIO-RL Platelets From a Previous Study (FerideX I.V) AND THIS STUDY (PREPARED)

\begin{tabular}{|c|c|c|c|c|c|c|c|}
\hline SPIO type & Sample & $\begin{array}{c}\text { Fe loading } \\
\text { (fg Fe/platelet) }\end{array}$ & $\begin{array}{c}\text { High Field } \\
\text { Effective } \\
\text { Moment }\left(10^{3} \mu_{8}\right)\end{array}$ & $\begin{array}{c}\text { Low Field } \\
\text { Effective } \\
\text { Moment }\left(10^{3} \mu_{8}\right)\end{array}$ & $\begin{array}{c}\text { Predicted } \\
\text { Magnetization for } \\
\text { MMOCT experiment } \\
\text { (10 } 0^{-3} \text { emu/g sample) }\end{array}$ & $\begin{array}{l}\text { MMOCT signal } \\
\text { at } 67 \mu \mathrm{g} \mathrm{Fe} / \mathrm{mL} \\
\text { (dB) }\end{array}$ & $\begin{array}{c}\text { Platelet Number } \\
\text { Density at } 67 \mu \mathrm{g} \\
\mathrm{Fe} / \mathrm{mL}\end{array}$ \\
\hline Feridex & Free SPIO & - & $10 \pm 2$ & - & $3.2 \pm 0.2$ & $3.3 \pm 0.6$ & - \\
\hline Feridex & SPIO-RL platelets & $48.3 \pm 0.6$ & $10 \pm 2$ & - & $3.1 \pm 0.2$ & $1.6 \pm 0.2$ & $1.5 \times 10^{9} / \mathrm{cm}^{3}$ \\
\hline prepared & Free SPIO & - & $7.3 \pm 0.6$ & $12 \pm 2$ & $1.7 \pm 0.5$ & $0.98 \pm 0.07$ & - \\
\hline prepared & SPIO-RL platelets & $216 \pm 17$ & $27 \pm 3$ & $43 \pm 6$ & $8.0 \pm 3.0$ & $4.5 \pm 0.4$ & $0.31 \times 10^{9} / \mathrm{cm}^{3}$ \\
\hline
\end{tabular}

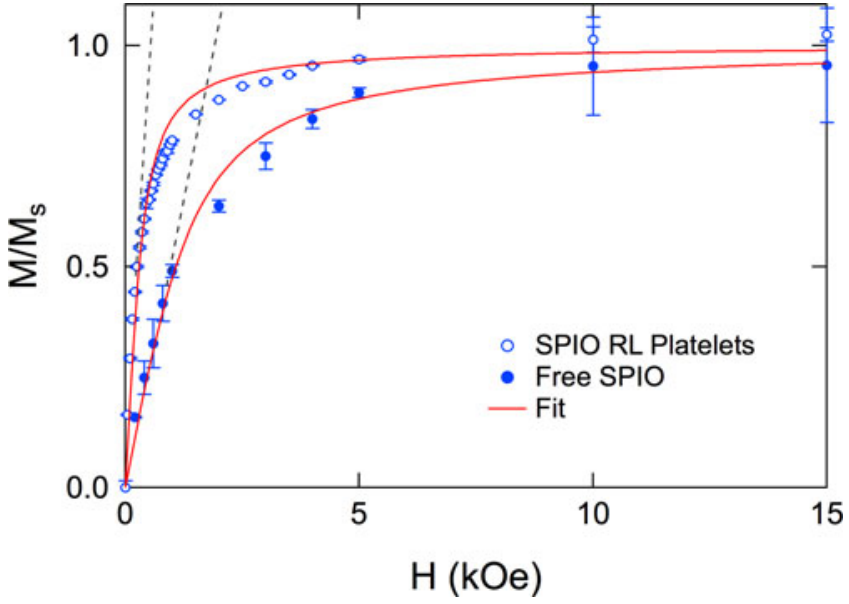

Fig. 4. Representative magnetization $M$ versus applied magnetic field $H$ data for freely dispersed SPIOs and SPIO-RL platelet aggregates at $20^{\circ} \mathrm{C}$, with best-fit line according to (4). Magnetization is normalized by the saturation magnetization $M_{s}$ for each sample. The apparent systematic difference between theory and experiment is possibly due to size polydispersity of the SPIOs resulting in a distribution of $\mu$ values. The dashed lines are extensions of the low-field slopes as a visual guide.

then, is one that provides the greatest magnetization at fields below saturation. Functionally, this figure of merit is improved by either increasing the total amount of Fe inside each platelet or increasing the effective size of each superparamagnetic spin cluster, i.e., effective moment $\mu$. As we show below, the SPIORL platelets aggregates in this study display improvements in both of these.

As described in (4), this effective superparamagnetic spin cluster $\mu$ directly affects the susceptibility and thus the initial slope of the magnetization at low field, as shown in Fig. 4, where example field-dependent magnetization curves for free SPIOs and SPIO-RL platelets are plotted. The SPIO-RL platelets in this study are found to display an effective spin cluster $>3 \times$ larger than free SPIOs (see Table I); accordingly, the initial slope of these samples is much larger ( $\sim 3-4$ times) than that for free SPIOs (see Fig. 4). While $M_{s}$ is proportional to the total mass of $\mathrm{Fe}$, the normalized low-field slope and $\mu$ are independent of the Fe loading of the SPIO-RL platelets.

As a separate measurement of $\mu$, temperature-dependent magnetization and susceptibility at low applied fields were obtained and fit to the Curie law in (5) (see Fig. 5). Because these fields
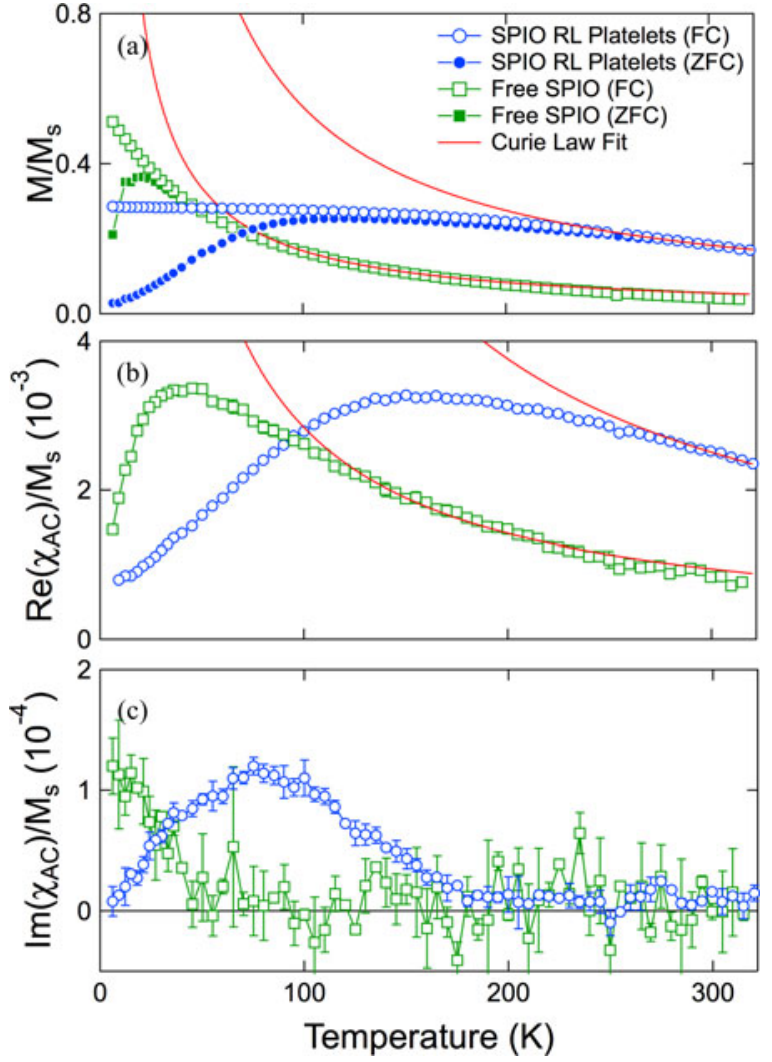

Fig. 5. Temperature-dependent FC and ZFC magnetometry of SPIOs and SPIO-RL platelet aggregates, with a Curie law fit according to (5). The magnetization $M$ is normalized by the saturation magnetization $M_{s}$ for each sample. (a) DC magnetometry at $50 \mathrm{Oe}$; (b) and (c) real and imaginary parts, respectively, of the ac magnetic susceptibility $\left(\chi_{\mathrm{AC}}\right)$ at $10 \mathrm{~Hz}$ and $4 \mathrm{Oe}$.

(50-Oe dc field and 4-Oe ac modulations) are lower than those used in the field-dependent scans, the values obtained are expected to be very close to the zero-field value of $\mu$. As seen in Table I, $\mu$ from the temperature-dependent (low-field) scans are higher than that from the field-dependent (high-field) scans, but show the same relative enhancement for that of SPIO-RL platelets compared to that of the free SPIOs.

The enhancement in $\mu$ for the SPIO-RL platelet aggregates from free SPIOs was not observed for the SPIO-RL platelets in our previous study with Feridex I.V., where the values for $\mu$ are the same for both and only slightly larger than that of the prepared, free SPIOs in this study. Interestingly, this apparent 
increase in the effective magnetic cluster size for the SPIO-RL platelet aggregates is associated with a larger Fe loading, as well as an aggregated, clot-like nature. This suggests that magnetic interactions between SPIO particles are becoming significant, similar to other reports in tightly packed SPIO systems [33].

In addition, the characteristic temperatures, e.g., blocking temperature, associated with low-temperature superparamagnetic behavior also exhibit corresponding large enhancements, as shown in Fig 5. The measured dc magnetization exhibits FC and ZFC irreversibility with a characteristic ZFC maximum below the onset of irreversibility [see Fig. 5(a)]. Both the irreversibility temperature and the $\mathrm{ZFC}$ maximum temperature show enhancements for the SPIO-RL platelet aggregates with respect to the free SPIOs. At temperatures above the onset of irreversibility, the samples are superparamagnetic, as both the $\mathrm{dc}$ behavior and the real part of ac susceptibility (in-phase component) obey the Curie law (5) [lines in Fig. 5(a) and (b)]. From the Curie law fits, the low-field values for $\mu$ were obtained (see Table I). At lower temperatures, the real part of the ac susceptibility (in-phase component) exhibits a maximum, which corresponds to the blocking temperature at the measurement frequency. The respective blocking temperatures for the SPIO-RL platelet aggregates and the free SPIOs are $150 \pm 5 \mathrm{~K}$ and $50 \pm 5 \mathrm{~K}$. The latter is consistent with the literature value of $\sim 60 \mathrm{~K}$ for ferumoxides [34]. Below the blocking temperature, dissipation processes become significant as the imaginary part (out-of-phase component) of susceptibility becomes appreciable.

While the peaks in the ac susceptibility (both real and imaginary parts in Fig. 5(b) and (c), respectively) for the SPIO-RL platelet aggregates shift to higher temperatures from those of the free SPIOs, the normalized peak heights are nearly identical between the two types of samples. This observation indicates that, other than having a different effective size of superparamagnetic spins, interactions between them are "free SPIO-like," i.e., negligible. One possible scenario emerges, such that within the platelet aggregates, 3-4 SPIO particles form tightly packed clusters with sufficiently strong interparticle magnetic interactions to couple their spins together, leading to a larger effective superparamagnetic spin. The nature of the small-packed clusters and the mechanism for their formation are presently unknown; elucidating these would require further investigations.

In order to relate these magnetic property measurements to MMOCT contrast, MMOCT was performed on free SPIOs and SPIO-RL platelet aggregates dispersed in $1 \%$ agarose with $0.5-$ $2 \mathrm{mg} / \mathrm{g} \mathrm{TiO}_{2}$ micropowder as an optical scatterer. The measured magnetic properties of each sample were used to predict the induced magnetization at the peak modulation magnetic field of 0.15 T used during MMOCT (see Table I), while accounting for the negative magnetization from the diamagnetic background. In order to compare these samples with those measured previously, the Fe concentration was held constant at $67 \mu \mathrm{g} / \mathrm{mL}$.

As expected, the predicted magnetization for each sample is strongly correlated with the resulting MMOCT contrast in tissue phantoms (see Table I). Representative MMOCT images of prepared SPIOs and SPIO-RL platelet aggregates are displayed in Fig. 6. The highest contrast at $67 \mu \mathrm{g} / \mathrm{mL}$ was obtained for the

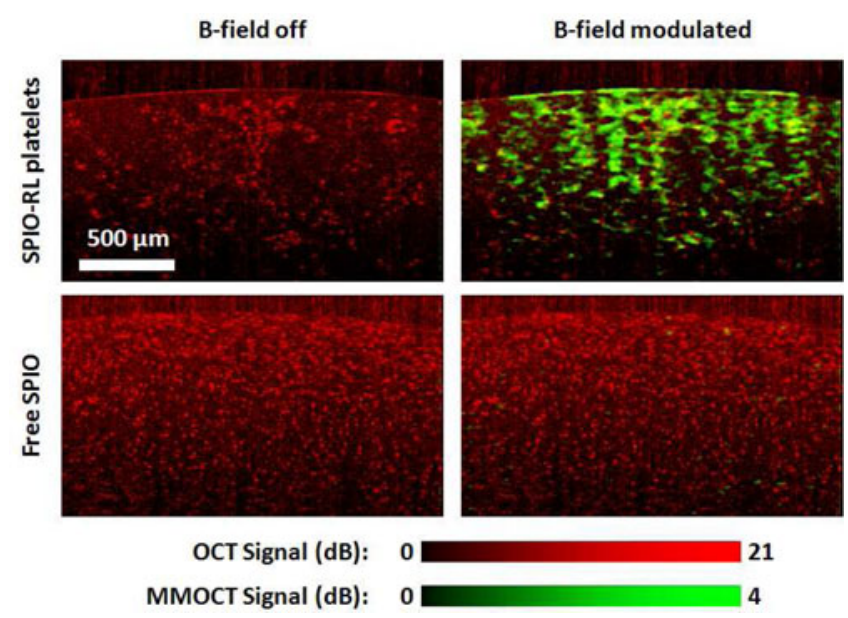

Fig. 6. OCT (red) and MMOCT (green) of agarose tissue phantoms containing free SPIOs and SPIO-RL platelet aggregates at $67 \mu \mathrm{g} \mathrm{Fe} / \mathrm{mL}$. While a small amount of signal is observed for free SPIO, MMOCT contrast is significantly enhanced in the aggregated platelets.

SPIO-RL platelet aggregates, averaging $4.5 \pm 0.4 \mathrm{~dB}$. Importantly, the platelet number density this corresponds to is only $0.31 \times 10^{9} / \mathrm{cm}^{3}$, or over $4 \times$ less than the previous SPIO-RL platelets.

These results suggest that the improved MMOCT contrast properties of SPIO-RL platelet aggregates are both a function of the improved Fe loading, and of particle-particle magnetic interactions, which improve their magnetic properties at the fields used in MMOCT imaging. Future study is needed to determine whether the aggregation of the platelets into a clot-like formation causes some of these improved properties, which may be a new mechanism of enhancing contrast in vivo that is activated when SPIO-RL platelets adhere to clots.

\section{MMOCE of Simulated Clots is Related to Fibrin Content}

As a proof of principle, we show how the use of magnetic forces and OCT may also be used to assess the stiffness of clots. Here, we use purified fibrin clots produced with varying amounts of fibrinogen, which is an important component of both arterial and venous thrombi. Fibrin network properties (viscoelasticity, permeability, and resistance to lysis) have been correlated with bleeding and clotting disorders [35]. Furthermore, fibrin network structure (branch point and fiber densities) is known to significantly influence the clot elastic modulus [36], [37].

Using MMOCE, we measured the acoustic spectrum of fibrin clots polymerized into open-faced rectangular wells. Similar to previous work with agarose phantoms [11], we found that the fundamental resonance frequency, $f_{0}=\omega_{0} / 2 \pi$, could be measured by fitting the spectra of the mechanical amplitude and phase lag (the absolute value and argument of $\tilde{A}(\omega)$, respectively), to (3). A representative example is shown in Fig. 7. In this example, the fundamental mode near $105 \mathrm{~Hz}$ is slightly overlapped by a higher frequency mode near $175 \mathrm{~Hz}$, which reduces the goodness of fit at frequencies above the fundamental mode. In particular, the phase lag shifts from 0 (in-phase motion) to $\pi$ (out-of-phase motion) as the frequency is swept past the 

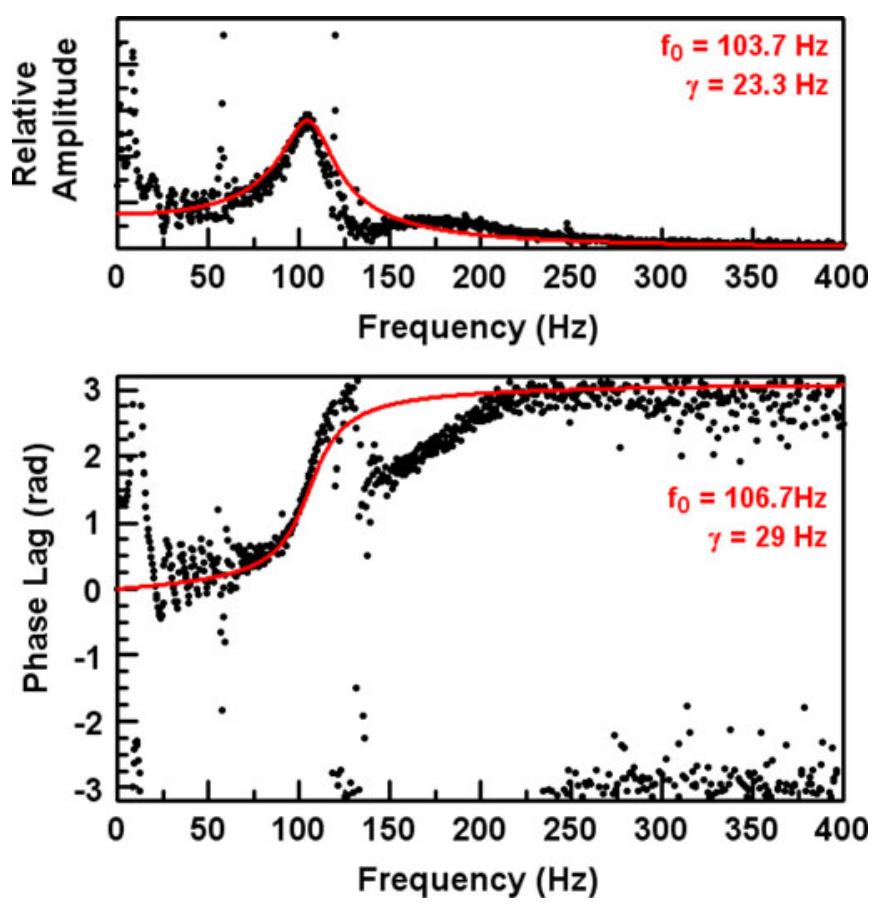

Fig. 7. Acoustic spectral amplitude and phase lag of a fibrin clot made with $5 \mathrm{mg} / \mathrm{mL}$ fibrinogen showing a fundamental resonance mode near $105 \mathrm{~Hz}$, and a second mode near $175 \mathrm{~Hz}$. Best fit curves are plotted in red for the fundamental mode according to (3).

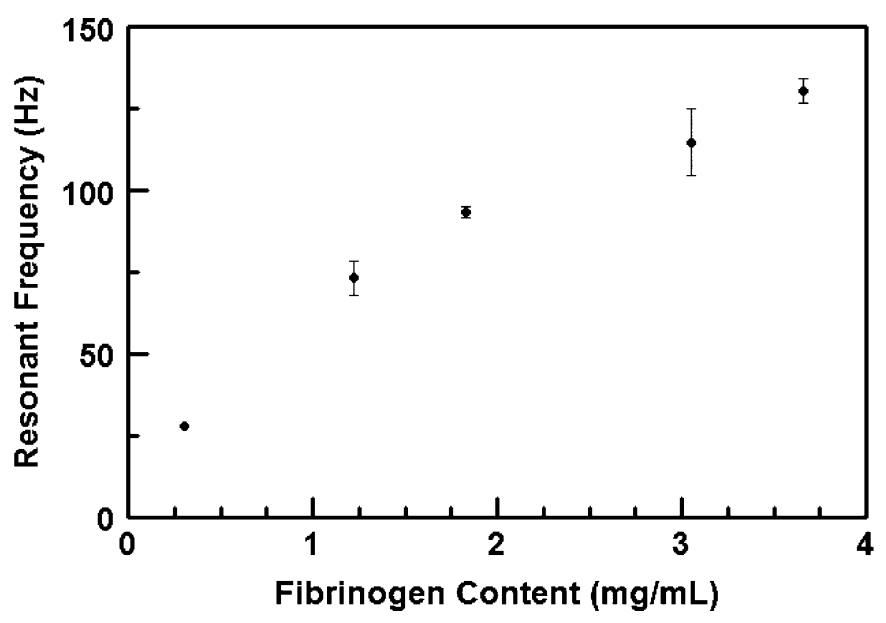

Fig. 8. Plot of the resonant frequency of the longitudinal mode in fibrin clots as a function of the fibrinogen concentration. Elastic modulus is proportional to the square of the resonant frequency.

resonance. Because the phase spectrum shows the phase lowering again immediately after reaching $\pi$ above $f_{0}$, it is indicative of a second mechanical mode being excited near $175 \mathrm{~Hz}$. Also, noise peaks are evident at $60 \mathrm{~Hz}$ and its harmonics. Despite these difficulties, it was possible to fit both curves to a consistent value of $f_{0}$ (103.7 Hz for the amplitude and $106.7 \mathrm{~Hz}$ for the phase). Furthermore, the fibrin clot system appears to be underdamped, with a quality factor $Q \approx 4$.

We found that the fundamental-mode frequency for fibrin clots was highly correlated with fibrinogen content (see Fig. 8).
Because the clots are all polymerized into wells with the same dimensions and boundary conditions, we know that the elastic modulus $E$ is proportional to $f_{0}^{2}$ according to (2). Therefore, the apparent $4.7 \times$ increase in $f_{0}$ from 0.3 to $3.7 \mathrm{mg} / \mathrm{mL}$ is indicative of a $22 \times$ increase in the clot elastic modulus. This finding is similar to previous fibrin-dependent measurements of the clot elastic modulus [37]. However, unlike previous techniques, this method has the advantage of being a resonance method that has the potential for high accuracy. The high displacement sensitivity of the OCT system provides typically microstrain sensitivity, permitting the evaluation of the clot elastic modulus under perturbative strain conditions where the behavior is linear, reversible, and easy to model. Furthermore, the use of the same MMOCT/MMOCE system hardware for both clot imaging and elastometry can lead to new associations between clot structure and function.

\section{CONCLUSION}

Our findings show how MMOCT/MMOCE can be used as a platform for both imaging and elastometry of blood clots. Evidence of particle-particle magnetic interactions suggests that SPIOs inside RL platelets under aggregated conditions, such as those found in a blood clot, can provide enhanced contrast properties for MMOCT and lower the threshold for detection to a clot loading of $3.1 \times 10^{8}$ platelets $/ \mathrm{cm}^{3}$. At the same time, MMOCE reveals the strong fibrinogen dependence of clot elastic modulus. As it stands, this MMOCT/MMOCE platform has uses for fundamental studies of blood clot formation dynamics, and may uniquely be used to connect clot viscoelastic properties with platelet activity, and to assess the efficacy of RL platelets as a hemostatic therapy.

Future work may lead to methods for the in vivo detection and assessment of clot elastic modulus by using SPIO-RL platelets as both the imaging agent, and as the transducer for acquiring the acoustic spectrum of the clot. This will pose several challenges. The arbitrary shape of blood clots will require imaging of clot boundary conditions and subsequent construction of finite element models to associate acoustic vibrations with mechanical properties. The physiological motion of the blood vessel due to cardiac function will need to be rejected, possibly by bandpass filtering as performed for MMOCT imaging in [8], or by appropriate synchronization of the chirped scan with the cardiac cycle. In the long term, an intravascular MMOCT/MMOCE system may be envisioned in which magnetic gradient fields are delivered from outside the patient while employing a standard OCT catheter for imaging. In the more immediate future, in vivo imaging of superficial vessels in animal models of vascular damage will guide the design of an intravascular imaging system.

By leveraging the ability for SPIO-RL platelets to target blood clots, we may envision an MMOCT/MMOCE device that can both detect early stage thrombosis and assess its relative risk to the patient by viscoelastic property measurement. Fundamentally, the ability to image nanoscale displacements induced by 
magnetic manipulation of SPIOs in tissue and loaded into cells will lead to many new biophysical discoveries.

\section{ACKNOWLEDGMENT}

The authors would like to thank R. K. Chhetri for his technical assistance with the construction and operation of the OCT system, and T. C. Nichols for providing porcine blood for magnetic analysis, both of whom are at the University of North Carolina at Chapel Hill.

\section{REFERENCES}

[1] I. K. Jang, B. E. Bouma, D. H. Kang, S. J. Park, S. W. Park, K. B. Seung, K. B. Choi, M. Shishkov, K. Schlendorf, E. Pomerantsez, S. L. Houser, H. T. Aretz, and G. J. Tearney, "Visualization of coronary atherosclerotic plaques in patients using optical coherence tomography: Comparison with intravascular ultrasound," J. Am. Coll. Cardiol., vol. 39, pp. 604-609, 2002.

[2] M. E. Brezinski, "Optical coherence tomography for identifying unstable coronary plaque," Int. J. Cardiol., vol. 107, pp. 154-165, 2006.

[3] G. J. Tearney, I.-K. Jang, and B. E. Bouma, "Imaging coronary atherosclerosis and vulnerable plaques with optical coherence tomography," in $O p$ tical Coherence Tomography: Technology and Applications, W. Drexler and J. G. Fujimoto, Eds. Berlin, Germany: Springer, 2007, ch. 34.

[4] N. Burris, K. Schwartz, C.-M. Tang, M. S. Jarfi, J. Schmitt, M. H. Kwon, O. Toshinaga, J. Gu, J. Brown, E. Brown, R. Pierson, and R. Poston, "Catheter-based infrared light scanner as a tool to assess conduit quality in coronary artery bypass surgery," J. Thorac. Cardiovasc. Surg., vol. 133, pp. 419-427, 2007.

[5] H. Azarnoush, S. Vergole, R. Bourezak, B. Boulet, and G. Lamouche, "Optical coherence tomography monitoring of angioplasty balloon inflation in a deployment tester," Rev. Sci. Instr., vol. 81, pp. 083101-1-083101-8, 2010.

[6] A. L. Oldenburg, J. R. Gunther, and S. A. Boppart, "Imaging magnetically labeled cells with magnetomotive optical coherence tomography," Opt. Lett., vol. 30, pp. 747-749, 2005.

[7] A. L. Oldenburg, F. Jean-Jacques Toublan, K. S. Suslick, A. Wei, and S. A. Boppart, "Magnetomotive contrast for in vivo optical coherence tomography," Opt. Exp., vol. 13, pp. 6597-6614, 2005.

[8] A. L. Oldenburg, V. Crecea, and S. A. Boppart, "Phase resolved magnetomotive OCT for imaging nanomolar concentrations of magnetic nanoparticles in tissues," Opt. Exp., vol. 16, pp. 11525-11539, 2008.

[9] R. John, R. Rezaeipoor, S. G. Adie, E. J. Chaney, A. L. Oldenburg, M. Marjanovic, J. P. Haldar, B. Sutton, and S. A. Boppart, "In vivo magnetomotive optical molecular imaging using targeted magnetic nanoprobes," Proc. Natl. Acad. Sci., vol. 107, pp. 8085-8090, 2010.

[10] V. Crecea, A. L. Oldenburg, X. Liang, T. S. Ralston, and S. A Boppart, "Magnetomotive nanoparticle transducers for optical rheology of viscoelastic materials," Opt. Exp., vol. 17, pp. 23114-23122, 2009.

[11] A. L. Oldenburg and S. A. Boppart, "Resonant acoustic spectroscopy of soft tissues using embedded magnetomotive nanotransducers and optical coherence tomography," Phys. Med. Biol., vol. 55, pp. 1189-1201, 2010.

[12] K. Jurk and B. E. Kehrel, "Platelets: Physiology and biochemistry,” Semin. Thromb. Hemost., vol. 31, pp. 381-392, 2005.

[13] M. S. Read, R. L. Reddick, and K. M. Brinkhous, "Preservation of hemostatic and structural properties of rehydrated lyophilized platelets: Potential for long-term storage of dried platelets for transfusion," Proc. Natl. Acad. Sci., vol. 92, pp. 397-401, 1995.

[14] A. L. Oldenburg, C. M. Gallippi, F. Tsui, T. C. Nichols, K. N. Beicker, R. K. Chhetri, D. Spivak, A. Richardson, and T. H. Fischer, "Magnetic and contrast properties of labeled platelets for magnetomotive optical coherence tomography," Biophys. J., vol. 99, pp. 2374-2383, 2010.

[15] K. Fatah, A. Silveira, P. Tornvall, F. Karpe, M. Blomback, and A. Hamsten, "Proneness to formation of tight and rigid fibrin gel structures in men with myocardial infarction at a young age," Thromb. Haemost., vol. 76 , pp. 535-540, 1996.

[16] J. P. Collett, Y. Allali, C. Lesty, M. L. Tanguy, J. Silvain, A. Ankri, B. Blanchet, R. Dumaine, J. Gianetti, L. Payot, J. W. Weisel, and
G. Montalescot, "Altered fibrin architecture is associated with hypofibrinolysis and premature coronary atherothrombosis," Arterioscler. Thromb. Vasc. Biol., vol. 26, pp. 2567-2573, 2006.

[17] A. Undas, P. Podolec, K. Zawilska, M. Pieculewicz, I. Jedlinski, E. Stepien, E. Konarska-Kuszewska, P. Weglarz, M. Duszynska, E. Hanschke, T. Przewlocki, and W. Tracz, "Altered fibrin clot structure/function in patients with cryptogenic ischemic stroke," Stroke, vol. 40, pp. 1499-1501, 2009.

[18] A. Undas, K. Zawilska, M. Ciesla-Dul, A. Lehmann-Kopydlowska, A. Skubiszak, K. Ciepluch, and W. Tracz, "Altered fibrin clot structure/function in patients with idiopathic venous thromboembolism and in their relatives," Blood, vol. 114, pp. 4272-4278, 2009.

[19] A. Undas, I. Wiek, E. Stepien, K. Zmudka, and W. Tracz, "Hyperglycemia is associated with enhanced thrombin formation, platelet activation, and fibrin clot resistance to lysis in patients with acute coronary syndrome," Diab. Care, vol. 31, pp. 1590-1595, 2008.

[20] M. E. Carr, "Development of platelet contractile force as a research and clinical measure of platelet function," Cell Biochem. Biophys., vol. 38, pp. 55-78, 2003.

[21] D. Huang, E. A. Swanson, C. P. Lin, J. S. Schuman, W. G. Stinson, W. Chang, M. R. Hee, T. Flotte, K. Gregory, C. A. Puliafito, and J. G. Fujimoto, "Optical coherence tomography," Science, vol. 254, pp. 1178$1181,1991$.

[22] A. Wax, C. Yang, R. R. Dasari, and M. S. Feld, "Path-length-resolved dynamic light scattering: modeling the transition from single to diffusive scattering," Appl. Opt., vol. 40, pp. 4222-4227, 2001.

[23] A. F. Fercher, C. K. Hitzenberger, G. Kamp, and S. Y. El-Zaiat, "Measurement of intraocular distances by backscattering spectral interferometry," Opt.Commun., vol. 117, pp. 43-48, 1995.

[24] M. A. Choma, A. K. Ellerbee, C. Yang, T. L. Creazzo, and J. A. Izatt, "Spectral-domain phase microscopy," Opt. Lett., vol. 30, pp. 1162-1164, 2005.

[25] A. E. H. Love, A Treatise on the Mathematical Theory of Elasticity. New York: Dover, 1944, pp. 289-291

[26] R. K. Chhetri, J. Carpenter, R. Superfine, S. H. Randell, and A. L. Oldenburg, "Magnetomotive optical coherence elastography for relating lung structure and function in Cystic Fibrosis," in Proc. Int. Soc. Opt. Eng., 2010, vol. 7554, pp. 755420-1-755420-10.

[27] R. S. Molday and D. MacKenzie, "Immunospecific ferromagnetic irondextran reagents for the labeling and magnetic separation of cells," $J$. Immunol. Methods, vol. 52, pp. 353-367, 1982.

[28] O. Clement, N. Siauve, C.-A. Cuenod, and G. Frija, "Liver imaging with ferumoxides (Feridex): Fundamentals, controversies, and practical aspects," Top. Magn. Reson. Imaging, vol. 9, pp. 167-182, 1998.

[29] T. H. Fischer and S. E. Eskridge, "Delivery of micro- and nanoparticles with blood platelets," PCT World Intellectual Property Organization, Geneva, Switzerland, Patent WO/2008/143 769, Nov. 27, 2008.

[30] G. Endler, A. Klimesch, H. Sunder-Plassmann, M. Schillinger, M. Exner, C. Mannhalter, N. Jordanova, G. Christ, R. Thalhammer, K. Huber, and R. Sunder-Plassmann, "Mean platelet volume is an independent risk factor for myocardial infarction but not for coronary artery disease," $B r . J$. Haematol., vol. 117, pp. 399-404, 2002.

[31] Y. Sato, K. Hatakeyama, Y. Yamashita, K. Marutsuka, A. Sumiyoshi, and Y. Asada, "Proportion of fibrin and platelets differs in thrombi on ruptured and eroded coronary atherosclerotic plaques in humans," Heart, vol. 91, pp. 526-530, 2005

[32] T. H. Fischer, A. P. Bode, B. R. Parker, K. E. Russell, D. E. Bender, J. K. Ramer, and M. S. Read, "Primary and secondary hemostatic functionalities of rehydrated, lyophilized platelets," Transfusion, vol. 46, pp. 1943-1950, 2006.

[33] D. Serantes, D. Baldomir, M. Pereiro, C. E. Hoppe, F. Rivadulla, and J. Rivas, "Nonmonotonic evolution of the blocking temperature in dispersions of superparamagnetic nanoparticles," Phys. Rev. B, vol. 82, pp. 134433-1-6, 2010

[34] C. W. Jung and P. Jacobs, "Physical and chemical properties of superparamagnetic iron oxide MR contrast agents: Ferumoxides, ferumoxtran, ferumoxsil," Magn. Reson. Imaging, vol. 13, pp. 661-674, 1995.

[35] A. S. Wolberg, "Thrombin generation and fibrin clot structure," Blood Rev, vol. 21, pp. 131-142, 2007.

[36] J. P. Collett, H. Shuman, R. E. Ledger, S. Lee, and J. W. Weisel, "The elasticity of an individual fibrin fiber in a clot," Proc. Natl. Acad. Sci., vol. 102, pp. 9133-9137, 2005.

[37] M. E. Carr and S. L. Carr, "Fibrin structure and concentration alter clot elastic modulus but do not alter platelet mediated force development," Blood Coag. Fibrin, vol. 6, pp. 79-86, 1995 


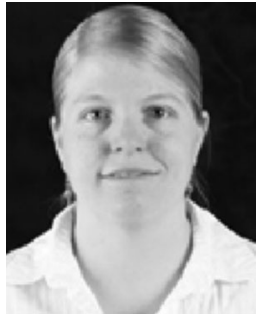

Amy L. Oldenburg was born in Berwyn, IL, in 1974. She received a diploma from the Illinois Mathematics and Science Academy, Aurora, in 1991, the B.S. (Hons.) degree in applied physics from the California Institute of Technology, Pasadena, in 1995, and the M.S. and Ph.D. degrees in physics from the University of Illinois at Urbana-Champaign, in 1997 and 2001, respectively.

During doctoral research, she studied alkali metal vapor dynamics with pulsed femtosecond lasers. Her postdoctoral work at the Beckman Institute for Advanced Science and Technology, University of Illinois at Urbana-Champaign, included developing novel molecular contrast imaging techniques and associated nanoparticles for use with optical coherence tomography. In 2008, she joined the Faculty at the University of North Carolina at Chapel Hill, where she is currently an Assistant Professor in the Department of Physics and Astronomy and an Adjunct Assistant Professor in the Department of Biomedical Engineering. She is also the Optical Imaging Modality Leader at the Biomedical Research Imaging Center. Her current research interests include the study of tissue viscoelastic properties using novel optical and acoustic coherence imaging techniques and nanoprobes.

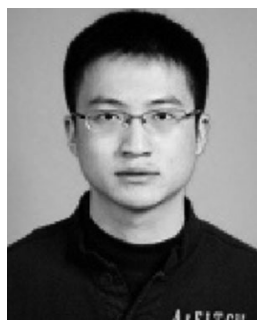

Gongting Wu was born in Jinhua, China, in 1987. He received the B.S degree in physics from Beijing Normal University, Beijing, China, in 2009. $\mathrm{He}$ is currently working toward the Ph.D. degree in physics at the University of North Carolina at Chapel Hill, under the supervision of Prof. A. Oldenburg.

His research interests include biodetection and biomechanical measurements using novel magnetomotive imaging.

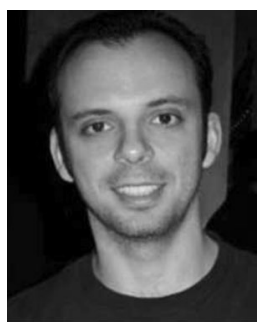

Dmitry Spivak was born in Moscow, Russia, in 1980, and then moved to America in 1992. He received the B.A. degree in physics from University of California at Berkeley, in 2002. In 2005, he received the M.S. degree in physics from the University of North Carolina at Chapel Hill, where he is currently working toward the Ph.D. degree in Prof. F. Tsui's lab.

His M.S. studies concentrated on building magnetically actuated nanoelectromechanical devices using single-walled carbon nanotubes. His doctoral research focuses on structural and electronic properties of novel magnetic materials, including magnetic semiconductors and half-metallic alloys.

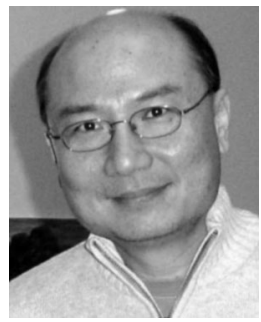

Frank Tsui received the B.S. (Hons.) degree in engineering physics from the University of California at Berkeley, Berkeley, in 1984, the M.Eng. degree in applied and engineering physics from Cornell University, Ithaca, NY, in 1986, and the M.S. and $\mathrm{Ph} . \mathrm{D}$. degrees in physics from the University of Illinois at Urbana-Champaign in 1987 and 1992, respectively.

He was a Margaret and Herman Sokol Postdoctoral Fellow in the Sciences at the University of Michigan at Ann Arbor, before he joined the Faculty of the University of North Carolina in 1995 as an Assistant Professor of Physics. He is currently a Professor of physics in the Department of Physics and Astronomy, University of North Carolina at Chapel Hill. His research interests include atomic scale synthesis and characterization of nanoscale magnetic materials, including novel synthesis of magnetic thin films and heterostructures using combinatorial molecular beam epitaxy techniques.

Dr. Tsui is a member of the American Physical Society. He was an IBM Predoctoral Fellow at the University of Illinois at Urbana-Champaign. He received the U.S. National Science Foundation CAREER Award in 1997.

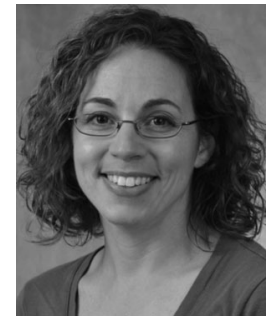

Alisa S. Wolberg received the B.S. and Ph.D. degrees from the University of North Carolina at Chapel Hill, in 1991 and 1996, respectively.

Her doctoral research focused on biochemical and structure/function relationships in human clotting factor IX. During her postdoctoral study at Duke University Medical Center, she investigated cellular regulation of tissue factor activity, procoagulant activity in human intravenous immunoglobulin preparations, and thrombin generation. She joined the Faculty in the Department of Pathology and Laboratory Medicine, University of North Carolina at Chapel Hill, in 2001, where she is currently an Associate Professor. Her current research interests include mechanisms connecting abnormal thrombin generation with hypo- and hypercoagulability, with particular focus on platelet activation and fibrin formation, structure, and stability.

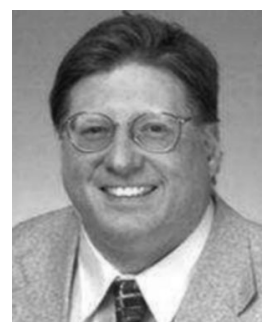

Thomas H. Fischer received the B.S. degree in physical chemistry from Florida State University, then worked with investigators at that same institution to complete his Ph.D. degree in biophysics in 1980. His primary $\mathrm{Ph} . \mathrm{D}$. research, which focused on the dynamic behavior of integral membrane protein-lipid complexes, was with Dr. T. P. Williams. In collaboration with Dr. P. M. Dirac and members of the Institute of Molecular Biophysics, he developed n-particle integral solutions of the Dirac equation for the analysis of electron behavior in molecular systems.

Subsequent to earning his Ph.D., he studied as a Postdoctoral Research Associate with Dr. C. Tanford at Duke University where he investigated the hydrophobic effect. He started his career in hemostasis in 1982 when he initiated work with Dr. G. C. White on problems related to intracellular signaling and cytoskeletal function in platelets. More recently, he has been involved with several biotechnology initiatives in the private sector. He has discovered a spray-drying process for dehydrating blood plasma for long-term storage in the developing world, and devised a glass-based bandage for control of topical hemorrhage. $\mathrm{He}$ is currently developing imaging therapeutics for the diagnosis and treatment of inflammation-based vascular disorders. He is the Scientific Director of the Francis Owen Blood Research Laboratory, a faculty member in the Department of Pathology and Laboratory Medicine, University of North Carolina at Chapel Hill, and the Chief Science Officer of Biolantic Partners, LLC. 